\title{
Web-based randomised controlled trials in orthodontics
}

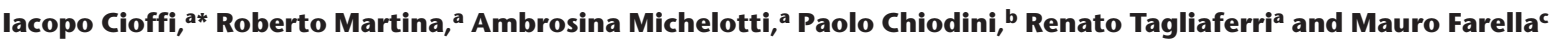 \\ ${ }^{a}$ Department of Orthodontics and Temporomandibular Disorders, University of Naples Federico II, ${ }^{b}$ Department of Medical Statistics, Second \\ University of Naples, Naples, Italy and ${ }^{c}$ Clinic for Masticatory Disorders and Complete Dentures, Centre for Oral Medicine, Dental and \\ Maxillofacial Surgery, University of Zurich, Zurich, Switzerland
}

\begin{abstract}
Randomised controlled trials (RCT) are considered the best source of scientific evidence - the gold standard - when evaluating the efficacy of orthodontic treatments. Frequently, RCT are planned as multicentre trials, with the intention of increasing statistical power and raising the precision of outcome estimates. The management of large-scale RCT, however, requires even more thorough organisation than conventional RCT. Indeed, the need for high accuracy and standardisation in data collection, research aids, secretarial skills, staff and patient training, and organisational meetings, make these studies time-consuming, expensive and, in general, relatively complex to carry out well.

A website was developed to support a large scale-orthodontic RCT which aimed to evaluate the efficacy of a functional appliance(www.ortodonzia.unina. it). Websites such as this can increase the quality of data collection, simplify the randomisation process, speed up data collection, and improve trial monitoring. Web-based RCT have the potential to help globalise orthodontic research and also increase our rate of acquisition of evidence in orthodontics.
\end{abstract}

Evidenced-based dentistry $(\mathrm{EBD})^{1}$ has a growing impact in orthodontics and, in the last decade, many researchers have performed clinical trials to test the efficacy and reliability of orthodontic procedures. ${ }^{2-4}$ Of the research tools available, RCT are still considered the best source of scientific evidence ${ }^{5-6}$ for evaluating the effects of dental and medical treatments. Frequently, RCT are planned as multicentre trials, so that statistical power and precision of outcome estimates can be increased (see the online publication "Randomised Controlled Trials. A User's Guide" by AR Jadad; www.cgmh.org.tw/intr/ intr5/c6700/OBGYN/F/Randomized\%20tial/ chapter4.html). The more complex needs and organisation of such multicentre RCT, however, make them difficult to conduct: indeed, a co-ordination centre is advocated ${ }^{7}$ to correctly manage different research units.

Today the internet is a widely accessible and appealing tool to help conduct RCT, with its great technological capabilities and its ease and low cost of use. The interactivity of a world-wide web tool has even been suggested to increase the compliance of users when conducting surveys. ${ }^{8}$ Recently, the

Corresponding author: Dr. Iacopo Cioffi Department of Orthodontics and Temporomandibular disorders University of Naples "Federico II" Via Pansini 5 , I-80131 NAPOLI, Italy HYPERLINK "mailto:" iacopo.cioffi@unina.it need for affordable and high-quality research has provoked clinicians to perform webbased clinical trials. Web facilities have been used for research protocol dissemination, randomisation, data collection and trial monitoring. ${ }^{9-12}$ Several studies have reported that web-based trials provide numerous benefits, by enhancing research-staff training, improving trial monitoring and administration, ${ }^{13}$ and noticeably reducing the inconvenience of large-scale trials. ${ }^{2,12-17}$ Although successful in many medical fields, however, the internet revolution still seems only to brush dental research and has even less impact on orthodontics. ${ }^{18}$

Recently we developed a website to support a large-scale orthodontic RCT that aimed to evaluate the efficacy of a functional appliance. Our intention was to develop a system able to facilitate data collection, increase data quality, enhance trial management and provide an efficient tool that could link and directly manage several research units, spread throughout Italy.

\section{Website}

A website was constructed, located at www. ortodonzia.unina.it (link to RCT Sander) on an independent university server. Hypertext mark-up language and Java scripts were used to build the web pages. Namo Web Editor 5.0 (SJ Namo, San Jose, California, USA), Adobe Photoshop (version 6.0; Adobe Systems,
San Jose, California, USA), PowerPoint (Microsoft, Redmond, Washington, USA), Jasc Paint Shop Pro (version 7.0; Corel, Ottawa, Canada) were used to make the graphical user interface. The website was optimised for Internet Explorer (version 5.0; Microsoft). Since the trial involved Italian orthodontic departments, web pages were written in Italian.

The website was structured into three areas: general information, patient management and a user's guide (Fig. 1). The general information provided details of the research project, including full descriptions of the entire research protocol and all the clinical procedures necessary for the trial in an online check-list. In this section, "all the things to do" (ie, recruitment phases, data collection, dental impressions, photographs etc) were fully described step-by-step. In addition, PowerPoint slides concerning the laboratory procedures used to build the functional appliance and clinical charts were available. The general information area was freely accessible.

The patient management area was structured for data entry and consulting. Once patients were judged eligible for the study, their data were entered in an online form developed for data capturing. In this area, dental and body measures were collected and digital records uploaded. In order to avoid missing data, dropdown menus and check boxes were extensively used. Finally, a custom-made Java script was developed to check mandatory and incorrect fields. The script was structured to stop the submission process if form fields were incomplete, blank or filled with inappropriate (qualitative data versus numerical data entry and vice versa) or incorrect details (date formats, metric and weight measurements). An example flash movie of the real-time data verification is available (www.ortodonzia.unina.it/chart. html).

The randomisation process was automatic and available online. After the first data entry, enrolled patients were randomly allocated to two different groups by means of a 
block randomisation. Custom-made online software was utilised to allow the stratifying factors, gender and research centre. Identifiable personal data of patients were not available online and subjects were given a five-digit identification. The access to this section was restricted to authenticated users and protected by encryption, remote firewalls and passwords.

Individual usernames and passwords were built using custom software and were provided to research unit coordinators. The access to data from patients recruited in each dental clinic was restricted to investigators belonging to the same research unit. The access from other research units was forbidden.

Data were transmitted to the national coordinator unit (University of Naples Federico II, Naples, Italy) and stored on a remote database. Records were then uploaded to the website in the same section. By browsing this area, authenticated users were also able to view patient data and the current status of research.

\section{Discussion}

The quality of orthodontic clinical studies has recently been the subject of discussion. Indeed, it has been suggested that, in order to obtain definitive answers concerning orthodontic, and in particular, functional therapies, ${ }^{19}$ the quality of orthodontic studies needs to be enhanced.

The collection of data and management of research represent crucial phases of clinical trials. The accuracy and the standardisation of data collection are, in particular, of fundamental importance while conducting surveys. Recently, electronic data capturing (EDC) systems have been introduced in dental research to enhance the quality of clinical studies. EDC may reduce the time it takes to collect data while also improving the quality of collected data. Recent reports confirm that the shifting from paper-based data collection to EDC has facilitated collection of data, ${ }^{20}$ centralising analysis and reducing both trial time and costs. ${ }^{15,21}$.

Of the different tools available for EDC (such as local electronic databases, clientserver technology and websites), web-based systems are especially promising because of the familiarity of so many people with websurfing. ${ }^{22}$ Websites provide numerous benefits to investigators. First of all is the more accurate data collection: the use of dropdown menus, check boxes and Java applets in webpages reduce data entry errors, typos and missing data. Next, since users make choices

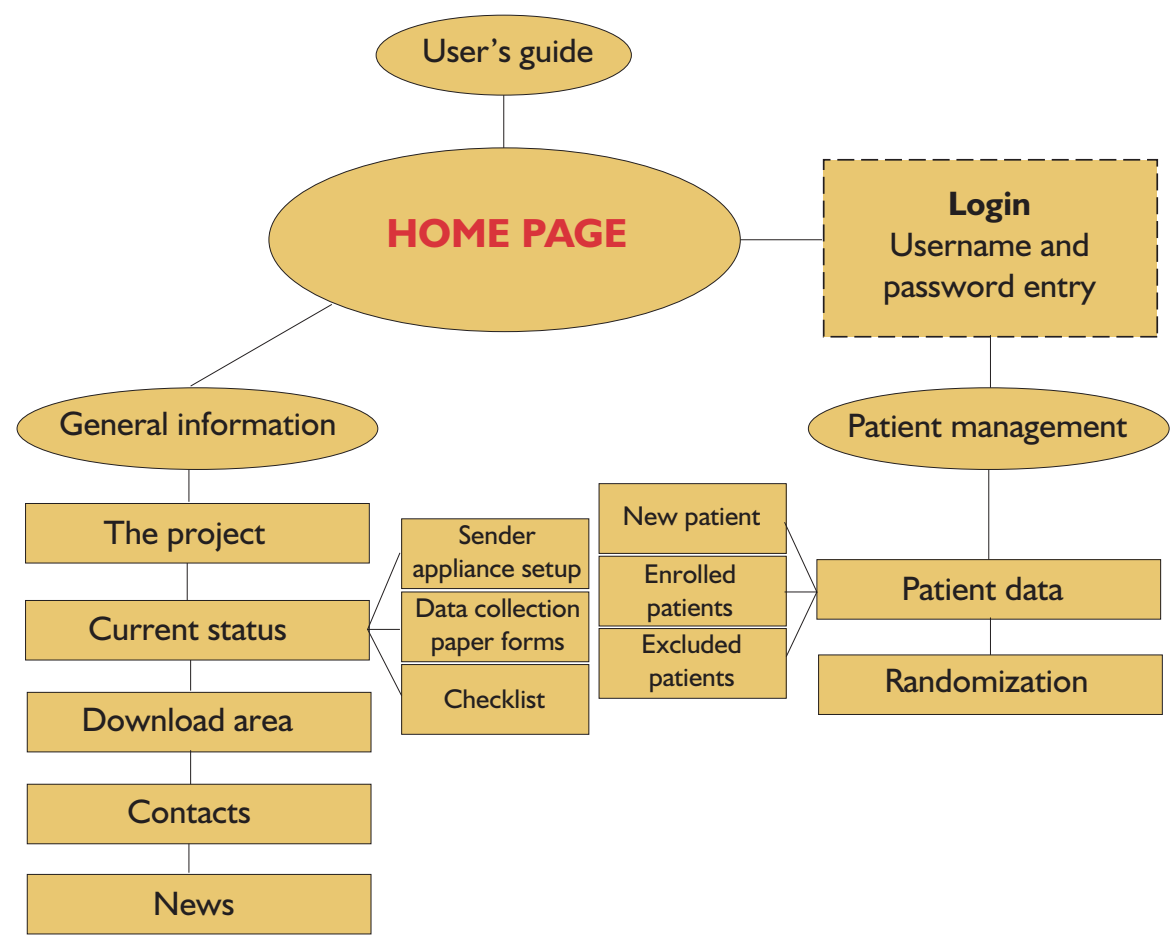

Figure 1. Structure of the website

from listed parameters in dropdown menus and click-check boxes, data collection can easily be standardised. Also, randomisation can be easily achieved online in real-time with custom software that randomly chooses subject allocation after first data entry.

Websites certainly allow faster data collection, and reduce the average time needed for carrying out RCT. With paper-based data collection, data were primarily collected on paper charts, and then, after a visual checking, were stored on local databases and sent to the co-ordination centre. With websites, an investigator only needs to log-in, insert the data of a patient, wait a second to let them be verified, and upload digital records. Moreover, these systems improve trial monitoring by directly managing several research units in real time. Scientific protocols, check lists, and informed consents for patients can be easily stored online, and may be downloaded within seconds. This, in turn, enhances trial administration, allowing researchers and clinicians to spend less time in administration and bureaucracy and more time focussing on patient health.

With respect to research-reporting, web facilities can be used to provide real-time and updated research information and facilitate the reporting of costs, while updated reports can be easily downloaded and printed from the remote database.

Finally, a central online database offers the opportunity to quickly expand studies to private dental offices and clinics. Feedback could be received by research units if online forums are used, to enhance the quality of the trial.

The web site, meanwhile, requires only a computer equipped with internet connection.

It is important to stress that use of such a database over the internet requires secure management and security policies. Continuous monitoring of network activity is mandatory, and encryption and firewalls are required to allow safe transactions and safe transfer of information. Cryptographic protocols similar to those used for online financial transactions might also be applied to web-based RCT. In general, several versions of protocols, such as Transport Layer Security Protocol and Secure Sockets Layer, can be used to provide security and secure data integrity for communications over $\mathrm{TCP} / \mathrm{IP}$ networks such as the internet. The use of these protocols along with a firewall prevents unauthorised accesses to computers located in a local or remote network. These systems regulate data transfers between computer networks by means 
of several trust levels defined by network administrator.

Finally, a user's guide was developed to aid clinicians in their use of the website, as well as detailing all the research and clinical procedures.

A few limitations of web-based facilities for research must be noted. Not all clinicians have internet connections in their dental offices, but tackling this problem is not difficult. The globalisation of mobile communication has provided cheap and efficient mobile internet connections for costumers. More importantly, because web tools must provide accurate data collection, comply with guidelines for correct data management, and be easy to use for temporary users, the cost of developing them may be considerable. To address this, dental societies and universities need to work together to develop flexible systems that can be customised to different research topics. Effort is still required within the web community to unify security policies for managing data over the internet: although criteria for data protection are quite similar in European countries, different policies are applied across other continents.

Finally, there is still a reluctance to shift from paper-based methods to electronically sourced documents in dental clinical trials. ${ }^{17}$ The solution to this is not technical features but lies with the motivation of researchers, who need to understand and accept the advantages of a new, easy-to-use, and fascinating method for their research.

\section{Conclusions}

The implementation of web-based RCT could increase the quality of data collection in orthodontics and enhance all phases of a clinical study. Remote data collection and validation by means of web-based tools is possible. Internet tools could also help coordinate research centres separated by great distances, and could enhance research management by improving personnel training, trial monitoring and patient care. Webbased research has the potential to help globalise and accelerate orthodontic and dental research. Although web tools have been extensively advocated in medical research to facilitate research procedures, we still lack the motivation of investigators to move from paper-based to web-based data collection: this change requires more than the offer of technical support for e-clinical research.

1. Rosenberg WC, Donald A. Evidence based medicine: an approach to clinical problem solving. Br Med J 1995; 310:1122-1126.

2. Ackerman M. Evidence-based orthodontics for the 21st century. J Am Dent Assoc 2004; 135:162-167.

3. Turpin DL. Evidence based orthodontics. Am J Orthod Dentofac Orthop 2000; 118:591.

4. Peck S. "Philosophy" and evidence-based orthodontics. Angle Orthod 1997; 67:403

5. Pocock SJ. Clinical Trials: a Practical Approach. Chichester: John Wiley; 1983.

6. Altman DG. Practical Statistics for Medical Research London: Chapman \& Hall; 1991.

7. Paul J,Seib R, Prescott T. The Internet and clinical trials: background, online resources, examples and issues. J Med Internet Res 2005; 7:e5.

8. Balter KA, Balter $O$, Fondell $E$, Lagerros YT. Webbased and mailed questionnaires: a comparison of response rates and compliance. Epidemiology 2005; 16:577-579.
9. Devineni T, Blanchard EB. A randomized controlled trial of an internet-based treatment for chronic headache. Behav Res Ther 2005; 43:277-292.

10. Kypri K, Sanders JB, Williams SM, McGee RO, Langley ID, Cashell-Smith ML. Web-based screening and brief intervention for hazardous drinking: a double blind randomized controlled trial. Addiction 2004; 99:1410-1417.

11. Dorman K, Saade GR, Smith H, Moise KJ. Use of the world wide web in research: randomization in a multicentre clinical trial of treatment for twintwin transfusion syndrome. Obstet Gynecol 2000; 96:636-639.

12. Santoro E, Nicolis E, Franzosi MG.

Telecommunication technology for the management of large scale clinical trials: the Gissi experience. Computer Methods Programs Biomed 1999; 60:215-223.

13. Marks RG. Validating electronic source data in clinical trials. Control Clin Trials 2004; 25:437-446.

14. Eysenbach $G$, Wyatt J. Using the Internet for surveys and health research. I Med Internet Res 2002; 4:e13.

15. McAlindon T, Formica M, Kabbara K, LaValley M, Lehmer M. Conducting clinical trials over the internet: feasibility study. Br Med J 2003; 327:484487.

16. Marks RG, Conlon M, Ruberg SJ. Paradigms shifts in clinical trials enabled by information technology. Stat Med 2001; 20:2683-2696.

17. Kelly MA, Oldham J. The Internet and randomised controlled trials. Int J Med Inform 1997; 47:91-99.

18. Marks RG. The future of web-based research in dentistry. J Dent Res 2004; 83 (Spec Iss C):25-C28.

19. O'Neill JR. Functional appliances and mandibular growth-is there an effect? Evid based Dent 2004; 5:74.

20. Meadows BJ. Eliciting remote data entry system requirements for the collection of cancer clinical trial data. Comput Inform Nurs 2003; 21:234-240.

21. Bart T. Comparison of electronic data capture with paper data collection - is there really an advantage? Business Briefing Pharmatech 2003:1-4.

22. Clivio L, Tinazzi A, Mangano S, Santoro E. The contribution of information technology: towards a better clinical data management. Drug Develop Res 2006; 67:245-250.

Evidence-Based Dentistry (2008) 9, 118-119. doi:10.1038/sj.ebd.640618 